\title{
Bäcklund transformations and divisor doubling
}

\author{
A.V. Tsiganov \\ St.Petersburg State University, St.Petersburg, Russia, Udmurt State University, Izhevsk, Russia \\ e-mail: andrey.tsiganov@gmail.com
}

\begin{abstract}
In classical mechanics well-known cryptographic algorithms and protocols can be very useful for construction canonical transformations preserving form of Hamiltonians. We consider application of a standard generic divisor doubling for construction of new auto Bäcklund transformations for the Lagrange top and Hénon-Heiles system separable in parabolic coordinates.
\end{abstract}

\section{Introduction}

The method of additive separation of variables in Hamilton-Jacobi equation, at least, in its most elementary forms such as separation of variables in elliptic, parabolic, spheroconical etc. coordinates, is a very important tool in analytical mechanics. Following to Jacobi works, an $n$-tuple $\left(H_{1}, \ldots, H_{n}\right)$ of functionally independent Hamiltonians in the involution will be said to be separable in a set of canonical coordinates $\left(x_{1}, \ldots, x_{n}, y_{1}, \ldots, y_{n}\right)$ if there are exist $n$ relations of the form

$$
\Phi_{i}\left(x_{i}, y_{i}, H_{1}, \ldots, H_{n}\right)=0, \quad i=1, \ldots, n, \quad \text { with } \quad \operatorname{det}\left[\frac{\partial \Phi_{i}}{\partial H_{j}}\right] \neq 0 .
$$

The reason for this definition is that the stationary Hamilton-Jacobi equations $H_{1}=\alpha_{1}, \ldots, H_{n}=\alpha_{n}$ can be collectively solved by the additively separated complete integral

$$
S\left(x_{1}, \ldots, x_{n} ; \alpha_{1}, \ldots, \alpha_{n}\right)=\sum_{i=1}^{n} S_{i}\left(x_{i} ; \alpha, \ldots, \alpha_{n}\right),
$$

where the $S_{i}$ are found by quadratures.

According to the Jacobi theorem if we substitute pairs of canonical coordinates $\left(x_{i}, y_{i}\right)$ into a system of separation relations

$$
\Phi_{i}\left(x, y, \alpha_{1}, \ldots, \alpha_{n}\right)=0, \quad i=1, \ldots, n, \quad \text { with } \quad \operatorname{det}\left[\frac{\partial \Phi_{i}}{\partial \alpha_{j}}\right] \neq 0 .
$$

and solve these equations with respect to $\alpha_{1}, \ldots, \alpha_{n}$, we obtain functionally independent Hamiltonians in the involution. Each separation relation $\Phi_{i}\left(x, y, \alpha_{1}, \ldots, \alpha_{n}\right)$ defines the curve $X_{i}$ on a $(x, y)$-plane, that allows us to consider integrable system $\left\{H_{1}, \ldots, H_{n}\right\}$ as an ordered set of points $\left\{P_{1}, \ldots, P_{n}\right\}$ on an ordered product $X_{i} \times \cdots \times X_{n}$.

For the systems separable in elliptic, parabolic, spheroconical etc. coordinate systems we have $n$ equivalent separation relations

$$
\Phi_{i}\left(x, y, \alpha_{1}, \ldots, \alpha_{n}\right)=\Phi_{j}\left(x, y, \alpha_{1}, \ldots, \alpha_{n}\right) \quad i, j=1, \ldots, n .
$$

If the corresponding plane curve $X$ has a necessary properties, we can consider formal sum of prime divisors $D=\sum_{i=1}^{n} P_{i}$ instead of an ordered set of points and identify this formal sum with a $n$-degree divisor $D$ on $X$ after compactification.

In hyperelliptic curve cryptography divisor $D$ on a hyperelliptic curve $X$ of genus $g$ plays the role of a message, which can be coding to a cryptogram $D^{\prime \prime}$, which is another divisor, using some standard cryptographic protocol. We can use secret protocols developed for full-degree divisors $n=g$ or degenerate divisors $n \neq g$, protocols based on divisor arithmetic or post quantum protocols based 
on isogenies etc, see [2, 3, 4, 10, 14, 23, and references within. Our main goal is to understand what means standard coding/decoding operations in classical mechanics and how to use these operations and the corresponding cryptograms in the theory of finite-dimensional integrable systems.

In this note we apply standard coding operations associated with addition and scalar multiplication of divisors

$$
D^{\prime \prime}=D+D^{\prime} \quad D^{\prime \prime}=[2] D
$$

to the divisors associated with Lagrange top and Hénon-Heiles system separable in parabolic coordinates. In cryptography divisor $D^{\prime}$ is a secret key, whereas scalar multiplication is a standard part of the keyless secret systems. We prove that in classical mechanics the corresponding coding operations are canonical transformations of valence one or two, which preserve the form of Hamilton-Jacobi equations. In [16, 17] reader could find another explicit formulae for canonical transformations for the Lagrange top and Hénon-Heiles system, which preserve the form of Hamiltonians, that determined our choice of examples. Canonical transformations preserving form of Hamiltonians we will call auto-Bäcklund transformations following to Toda and Wadati [24. There are also many other definitions of the autoBäcklund transformations for Hamilton-Jacobi equations [6, 12, 16, 22, but we prefer to use the oldest one.

\section{Divisor arithmetic on hyperelliptic curves}

Let us reproduce some definitions and facts from the following textbooks [5, 9].

A prime divisor on a smooth variety $X$ over a field $k$ is an irreducible closed subvariety $Z \subset X$ of codimension one, also defined over $k$.

Definition 1 A divisor is a finite formal linear combination

$$
D=\sum_{i} m_{i} Z_{i}, \quad m_{i} \in \mathbb{Z}
$$

of prime divisors. The group of divisors on $X$, which is the free group on the prime divisors, is denoted $\operatorname{Div} X$.

The group of divisors $\operatorname{Div} X$ is an additive abelian group under the formal addition rule

$$
\sum m_{i} Z_{i}+\sum n_{i} Z_{i}=\sum\left(m_{i}+n_{i}\right) Z_{i}
$$

To define an equivalence relation on divisors we use the rational functions on $X$. Function $f$ is a quotient of two polynomials; they are each zero only on a finite closed subset of codimension one in $X$, which is therefore the union of finitely many prime divisors. The difference of these two subsets define a principal divisor $\operatorname{div} f$ associated with function $f$. The subgroup of $\operatorname{Div} X$ consisting of the principal divisors is denoted by $\operatorname{Prin} X$.

Definition 2 Two divisors $D, D^{\prime} \in$ DivX are linearly equivalent

$$
D \approx D^{\prime}
$$

if their difference $D-D^{\prime}$ is principal divisor

$$
D-D^{\prime}=\operatorname{div}(f) \equiv 0 \quad \bmod \operatorname{Prin} X .
$$

The Picard group of $X$ is the quotient group

$$
\operatorname{Pic} X=\frac{\operatorname{Div} X}{\operatorname{Prin} X}=\frac{\text { Divisors defined over } \mathrm{k}}{\text { Divisors of functions defined over } \mathrm{k}} .
$$

For a general (not necessarily smooth) variety $\mathrm{X}$, what we have defined is not the Picard group, but the Weil divisor class group. For an irreducible normal variety $X$, the Picard group is isomorphic to the group of Cartier divisors modulo linear equivalence.

The Picard group is a group of divisors modulo principal divisors, and the group operation is formal addition modulo the equivalence relations. These group operations define so-called arithmetic of divisors in Picard group

$$
D+D^{\prime}=D^{\prime \prime} \quad \text { and } \quad[\ell] D=D^{\prime \prime},
$$


where $D, D^{\prime}$ and $D^{\prime \prime}$ are divisors, + and $[\ell]$ denote addition and scalar multiplication by an integer, respectively.

Let $X$ be a hyperelliptic curve of genus $g$ defined by equation

$$
y^{2}+h(x) y=f(x)
$$

where $f(x)$ is a monic polynomial of degree $2 g+2$ with distinct roots, $h(x)$ is a polynomial with $\operatorname{deg} h \leq g$. Prime divisors are rational point on $X$ denoted $P_{i}=\left(x_{i}, y_{i}\right)$, and $P_{\infty}$ is a point at infinity.

Definition 3 Divisor $D=\sum m_{i} P_{i}, m_{i} \in \mathbb{Z}$ is a formal sum of points on the curve, and degree of divisor $D$ is the sum $\sum m_{i}$ of the multiplicities of points in support of the divisor

$$
\operatorname{supp}\left(\sum m_{i} P_{i}\right)=\bigcup_{m_{i} \neq 0} P_{i}
$$

Quotient group of $\operatorname{Div} X$ by the group of principal divisors $\operatorname{Prin} X$ is called the divisor class group or Picard group. Restricting to degree zero, we can also define $\operatorname{Pic}^{0} X=\operatorname{Div}^{0} X / \operatorname{Prin} X$. The groups $\operatorname{Pic} X$ and $\operatorname{Pic}^{0} X$ carry essentially the same information on $X$, since we always have

$$
\operatorname{Pic} X / \operatorname{Pic}^{0} X \cong \operatorname{Div} X / \operatorname{Div}^{0} X \cong \mathbb{Z} .
$$

The divisor class group, where the elements are equivalence classes of degree zero divisors on $X$, is isomorphic to the Jacobian of $X$. By abuse of notation, a divisor and its class in Pic $X$ will usually be denoted by the same symbol.

In order to describe equivalence classes we can use semi-reduced and reduced divisors.

Definition 4 A semi-reduced divisor is divisor of the form

$$
D=\sum m_{i} P_{i}-\left(\sum m_{i}\right) P_{\infty},
$$

where $m_{i}>0, P_{i} \neq-P_{j}$ for $i \neq j$, no $P_{i}$ satisfying $P_{i}=-P_{i}$ appears more than once.

Because semi-reduced divisors are not unique in their equivalence class we introduce reduced divisors.

Definition 5 A semi-reduced divisor $D$ is called reduced if $\sum m_{i} \leq g$, i.e. if the sum of multiplicities is no more that genus of curve $C$. The reduced degree or weight of reduced divisor $D$ is defined as $w(D)=\sum m_{i}$.

This is a consequence of Riemann-Roch theorem for hyperelliptic curves that for each divisor $\tilde{D} \in$ $\operatorname{Div}^{0} X$ there is a unique reduced divisor $D$ so that $D \approx \tilde{D}$. For a thorough treatment see [5, 9 .

Using reduced divisors $D$ instead their equivalence classes we can describe fast and efficient algorithms for arithmetic on hyperelliptic curves. In 21] Mumford found polynomial representation of group elements $D=(u(x), v(x))$

$$
u(x)=\prod\left(x-x_{i}\right)^{m_{i}}, \quad v\left(x_{i}\right)=y_{i}, \quad \operatorname{deg}(V)<\operatorname{deg}(U) \leq g, \quad v^{2}-f \equiv 0 \bmod u .
$$

Here monic polynomial $u(x)$ may have multiple roots and $v(x)$ is tangent to the curve according to multiplicity roots. In fact these polynomials were introduced by Jacobi in the framework of the classical mechanics, see historical remarks, discussion and modern applications of these Jacobi polynomials in [13, 32.

In 2] Cantor proposed the following algorithm for performing arithmetic computations in Picard 
group of hyperelliptic curves $X$ defined by equation (2.2):

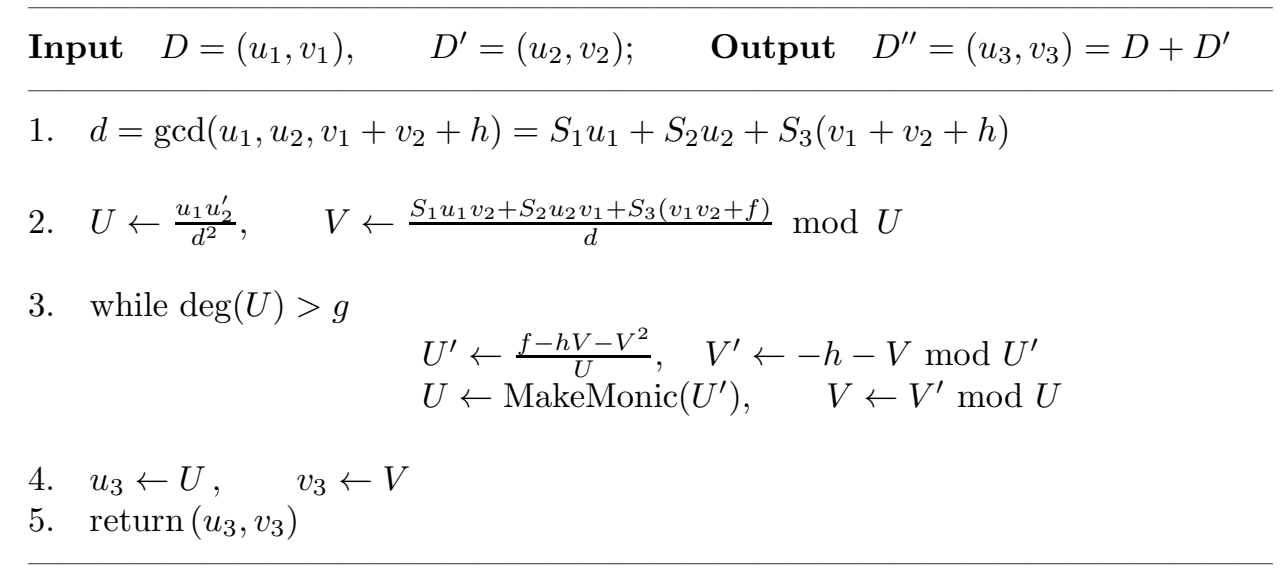

This algorithm consists of two stages: the composition stage, based on Gauss's classical composition of binary quadratic forms, which generally outputs an unreduced divisor with coordinates $(U, V)$, and the reduction stage, which transforms the unreduced divisor into the unique reduced divisor. The Cantor algorithm is quite slow due to its versatility and now we have a lot of other algorithms and their professional computer implementations for the divisor arithmetics.

\subsection{Arithmetic on genus 2 hyperelliptic curves}

For $g=2$ Cantors algorithm has since been substantially optimized in work initiated by Harley [11] , who was the first to obtain practical explicit formulas in genus two, and extended by Lange [19], who, among several others, generalized and significantly improved Harleys original approach, see dicsussion in 3 .

Let us present the Harley algorithm for addition and doubling:

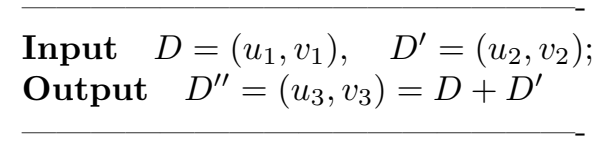

1. $U \leftarrow u_{1} u_{2}$

2. $S \leftarrow\left(v_{2}-v_{1}\right) / u_{1} \bmod u_{2}$

3. $V \leftarrow S u_{1}+v_{1} \bmod U$

4. $U \leftarrow\left(V^{2}+h V-f\right) / U$

5. Make $U$ monic

6. $V \leftarrow V \bmod U$

7. $u_{3} \leftarrow U, v_{3} \leftarrow-(V+h) \bmod u_{3}$

8. $\operatorname{return}\left(u_{3}, v_{3}\right)$

$$
\begin{aligned}
& \text { Input } \quad D=\left(u_{1}, v_{1}\right), \quad \operatorname{gcd}\left(u_{1}, h\right)=1 \\
& \text { Output } \quad D^{\prime \prime}=\left(u_{3}, v_{3}\right)=[2] D \\
& \hline \text { 1. } \quad U \leftarrow u_{1}^{2} \\
& \text { 2. } \quad S \leftarrow\left(2 v_{1}+h\right)^{-1}\left(f+h v_{1}-v_{1}^{2}\right) / u_{1} \bmod u_{1} \\
& \text { 3. } \quad V \leftarrow S u_{1}+v_{1} \bmod U \\
& \text { 4. } U \leftarrow\left(V^{2}+h V-f\right) / U \\
& \text { 5. } \quad \text { Make } U \operatorname{monic} \\
& \text { 6. } \quad V \leftarrow V \bmod U \\
& \text { 7. } u_{3} \leftarrow U, v_{3} \leftarrow-(V+h) \bmod u_{3} \\
& \text { 8. } \quad \text { return }\left(u_{3}, v_{3}\right)
\end{aligned}
$$

Composition parts from Step 1 to step 3 are based on the Chinese remainder theorem for addition and Newton iterations for doubling, respectively. We can take computer implementation of these algorithms from [11] and directly apply computer programs to divisors associated with integrable systems.

Let us also present well-known explicit formulae for these coding operations in the simplest case of the genus two hyperelliptic curve defined by equation (2.2) with $h(x)=0$

$$
X: \quad y^{2}=f(x), \quad f(x)=a_{5} x^{5}+a_{4} x^{4}+a_{3} x^{3}+a_{2} x^{2}+a_{1} x+a_{0} .
$$

Consider addition of full degree reduced divisor (message)

$$
D: \quad \operatorname{supp}(D)=\left\{P_{1}, P_{2}\right\} \cup\left\{P_{\infty}\right\}, w(D)=2
$$


with other reduced divisor $D^{\prime}$ (secret key) in the following cases

$$
\begin{aligned}
& \text { 1. } D+D^{\prime}=D^{\prime \prime}, \quad w\left(D^{\prime}\right)=2, \quad \operatorname{supp}\left(D^{\prime}\right)=\left\{P_{1}^{\prime}, P_{2}^{\prime}\right\} \cup\left\{P_{\infty}\right\} \text {, } \\
& \text { 2. }[2] D=D^{\prime \prime}, \quad D^{\prime}=D, \quad \operatorname{supp}\left(D^{\prime}\right)=\left\{P_{1}, P_{2}\right\} \cup\left\{P_{\infty}\right\}, \\
& \text { 3. } \quad D+D^{\prime}=D^{\prime \prime}, \quad w\left(D^{\prime}\right)=1, \quad \operatorname{supp}\left(D^{\prime}\right)=\left\{P_{1}^{\prime}\right\} \cup\left\{P_{\infty}\right\} \text {. }
\end{aligned}
$$

The result is full degree reduced divisor $D^{\prime \prime}$ (cryptogram) with $\operatorname{supp}\left(D^{\prime \prime}\right)=\left\{P_{1}^{\prime \prime}, P_{2}^{\prime \prime}\right\} \cup\left\{P_{\infty}\right\}$ and $w\left(D^{\prime \prime}\right)=2$.

In the second case operation

$$
[2] D=D+D=D^{\prime \prime}
$$

is called doubling of divisor $D$, i.e. scalar multiplication on integer $\ell=2$. Its inverse is called halving of $D^{\prime \prime}$ and for a given $D^{\prime \prime}$ equation (2.5) has $2^{2 g}$ solutions, any two of which differ by a 2-torsion divisor 8], for efficient implementation see 20] and references within. Below we do not consider halving, tripling and other operations because explicit formulae for the corresponding canonical transformations are quite bulky and unreadable. The third case in (2.4) corresponds to one-point auto Bäcklund transformations from [16, 22].

Let us consider intersection of $X$ with the second plane curve defined by equation

$$
y-\mathcal{P}(x)=0, \quad \mathcal{P}(x)=b_{3} x^{3}+b_{2} x^{2}+b_{1} x+b_{0} .
$$

in the framework of the standard intersection theory [1, 5, 15. Substituting $y=\mathcal{P}(x)$ into the equation (2.3), we obtain the so-called Abel polynomial [1]

$$
\psi(x)=\mathcal{P}(x)^{2}-f(x),
$$

which has no multiple roots in the first and third cases and has double roots in the second case:

$$
\begin{aligned}
& \text { 1. } \psi(x)=b_{3}^{2}\left(x-x_{1}\right)\left(x-x_{2}\right)\left(x-x_{1}^{\prime}\right)\left(x-x_{2}^{\prime}\right)\left(x-x_{1}^{\prime \prime}\right)\left(x-x_{2}^{\prime \prime}\right), \\
& \text { 2. } \psi(x)=b_{3}^{2}\left(x-x_{1}\right)^{2}\left(x-x_{1}^{\prime}\right)^{2}\left(x-x_{1}^{\prime \prime}\right)\left(x-x_{2}^{\prime \prime}\right), \\
& \text { 3. } \psi(x)=-a_{5}\left(x-x_{1}\right)\left(x-x_{2}\right)\left(x-x_{1}^{\prime}\right)\left(x-x_{1}^{\prime \prime}\right)\left(x-x_{2}^{\prime \prime}\right), \quad b_{3}=0 .
\end{aligned}
$$

The cases 1 and 2 are presented in Figure 1, which is standard Clebsch's geometric interpretation of the Abel results 15 .

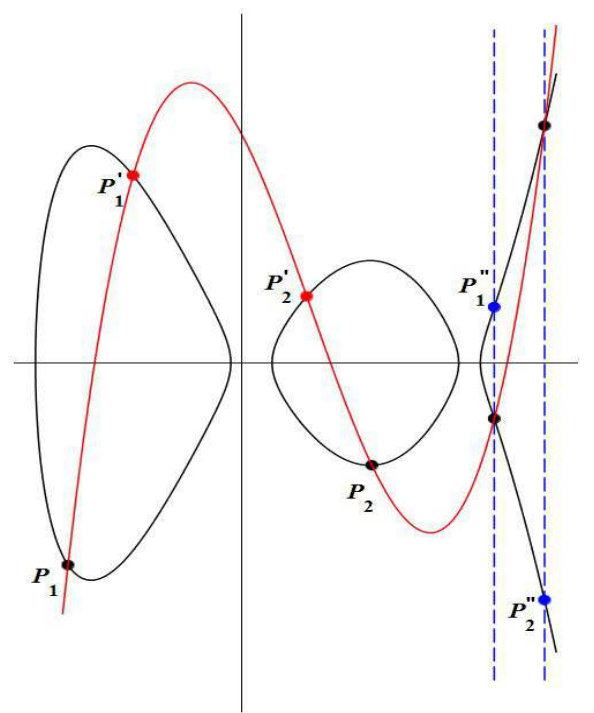

Case 1: $\left(P_{1}+P_{2}\right)+\left(P_{1}^{\prime}+P_{2}^{\prime}\right)=P_{1}^{\prime \prime}+P_{2}^{\prime \prime}$

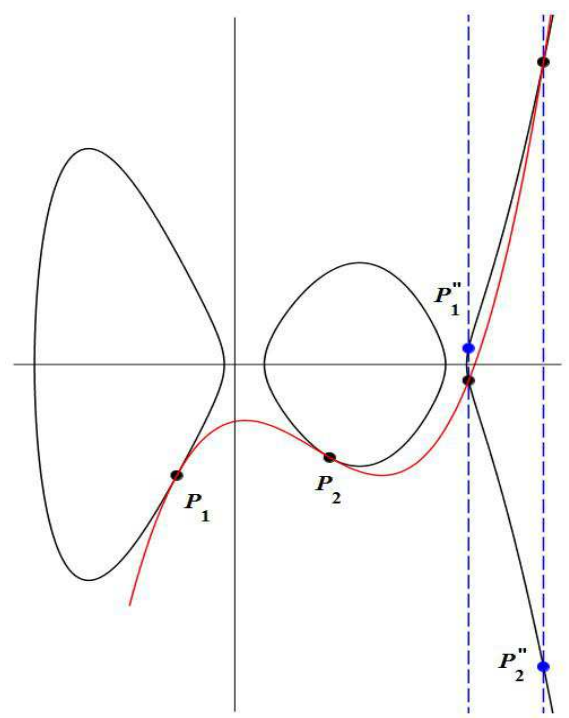

Case 2: $[2]\left(P_{1}+P_{2}\right)=P_{1}^{\prime \prime}+P_{2}^{\prime \prime}$ 
Equating coefficients of $\psi$ in the first case gives

$$
\begin{aligned}
& x_{1}^{\prime \prime}+x_{2}^{\prime \prime}=-x_{1}-x_{2}-x_{1}^{\prime}-x_{2}^{\prime}+\frac{a_{5}-2 b_{2} b_{3}}{b_{3}^{2}}, \\
& x_{1}^{\prime \prime} x_{2}^{\prime \prime}=\frac{2 b_{1} b_{3}+b_{2}^{2}-a_{4}}{b_{3}^{2}}-\left(x_{1}+x_{2}+x_{1}^{\prime}+x_{2}^{\prime}\right)\left(x_{1}^{\prime \prime}+x_{2}^{\prime \prime}\right)-x_{1}\left(x_{2}+x_{1}^{\prime}+x_{2}^{\prime}\right)-x_{2}\left(x_{1}^{\prime}+x_{2}^{\prime}\right)-x_{1}^{\prime} x_{2}^{\prime} .
\end{aligned}
$$

In the second case we have to put $x_{1,2}^{\prime}=x_{1,2}$ in these equations, whereas in the third case we have

$$
\begin{aligned}
& x_{1}^{\prime \prime}+x_{2}^{\prime \prime}=-x_{1}-x_{2}-x_{1}^{\prime}+\frac{b_{2}^{2}-a_{4}}{a_{5}}, \\
& x_{1}^{\prime \prime} x_{2}^{\prime \prime}=\frac{a_{3}-2 b_{1} b_{2}}{a_{5}}-\left(x_{1}+x_{2}+x_{1}^{\prime}\right)\left(x_{1}^{\prime \prime}+x_{2}^{\prime \prime}\right)-x_{1} x_{2}-x_{1} x_{1}^{\prime}-x_{2} x_{1}^{\prime} .
\end{aligned}
$$

Four coefficients $b_{3}, b_{2}, b_{1}$ and $b_{0}$ of polynomial $\mathcal{P}(x)(2.6)$ are calculated by solving four algebraic equations:

$$
\begin{aligned}
& \text { 1. } y_{1,2}=\mathcal{P}\left(x_{1,2}\right), \quad y_{1,2}^{\prime}=\mathcal{P}\left(x_{1,2}^{\prime}\right) ; \\
& \text { 2. } y_{1,2}=\mathcal{P}\left(x_{1,2}\right),\left.\quad \frac{d P(x)}{d x}\right|_{x=x_{1,2}}=\left.\frac{d \sqrt{f(x)}}{d x}\right|_{x=x_{1,2}} \equiv \frac{1}{2 y_{1,2}} \partial f\left(x_{1,2}\right), \\
& \text { 3. } y_{1,2}=\mathcal{P}\left(x_{1,2}\right), \quad y_{1}^{\prime}=\mathcal{P}\left(x_{1}^{\prime}\right), \quad b_{3}=0,
\end{aligned}
$$

where $\partial f(x)$ is derivative of $f(x)(2.3)$ by $x$. Substituting coefficients $b_{k}$ into (2.7) 2.8) one gets abscissas $\tilde{u}_{1,2}$, whereas the corresponding ordinates $y_{1,2}^{\prime \prime}$ are equal to

$$
y_{3,4}^{\prime \prime}=-\mathcal{P}\left(x_{1,2}^{\prime \prime}\right),
$$

where polynomial $\mathcal{P}(x)$ is given by

$$
\begin{aligned}
1 . \mathcal{P}(x) & =\frac{\left(x-x_{2}^{\prime}\right)\left(x-x_{1}^{\prime}\right)\left(x-x_{2}\right) y_{1}}{\left(x_{1}-x_{1}^{\prime}\right)\left(x_{1}-x_{2}^{\prime}\right)\left(x_{1}-x_{2}\right)}+\frac{\left(x-x_{2}^{\prime}\right)\left(x-x_{1}^{\prime}\right)\left(x-x_{1}\right) y_{2}}{\left(x_{2}-x_{1}^{\prime}\right)\left(x_{2}-x_{2}^{\prime}\right)\left(x_{1}-x_{2}\right)} \\
& +\frac{\left(x-x_{2}^{\prime}\right)\left(x-x_{2}\right)\left(x-x_{1}\right) y_{1}^{\prime}}{\left(x_{1}^{\prime}-x_{1}\right)\left(x_{1}^{\prime}-x_{2}\right)\left(x_{2}^{\prime}-x_{1}^{\prime}\right)}+\frac{\left(x-x_{1}^{\prime}\right)\left(x-x_{2}\right)\left(x-x_{1}\right) y_{2}^{\prime}}{\left(x_{2}^{\prime}-x_{1}\right)\left(x_{2}^{\prime}-x_{2}\right)\left(x_{2}^{\prime}-x_{1}^{\prime}\right)} \\
2 . \quad \mathcal{P}(x) & =\frac{\left(x-x_{2}\right)^{2}\left(2 x-3 x_{1}+x_{2}\right) y_{1}}{\left(x_{2}-x_{1}\right)^{3}}+\frac{\left(x-x_{1}\right)^{2}\left(2 x+x_{1}-3 x_{2}\right) y_{2}}{\left(x_{1}-x_{2}\right)^{3}} \\
& +\frac{\left(x-x_{2}\right)^{2}\left(x-x_{1}\right) \partial f\left(x_{1}\right)}{2\left(x_{1}-x_{2}\right)^{2} y_{1}}+\frac{\left(x-x_{1}\right)^{2}\left(x-x_{2}\right) \partial f\left(x_{2}\right)}{2\left(x_{1}-x_{2}\right)^{2} y_{2}}, \\
\text { 3. } \mathcal{P}(x) & =\frac{y_{1}\left(x-x_{2}\right)\left(x-x_{1}^{\prime}\right)}{\left(x_{1}-x_{2}\right)\left(x_{1}-x_{1}^{\prime}\right)}+\frac{y_{2}\left(x-x_{1}\right)\left(x-x_{1}^{\prime}\right)}{\left(x_{2}-x_{1}\right)\left(x_{2}-x_{1}^{\prime}\right)}+\frac{y_{1}^{\prime}\left(x-x_{1}\right)\left(x-x_{2}\right)}{\left(x_{1}^{\prime}-x_{1}\right)\left(x_{1}^{\prime}-x_{2}\right)} .
\end{aligned}
$$

In (2.10) we also made a reduction, which coincides with inversion $(x, y) \rightarrow(x,-y)$.

Similar formulae for the hyperelliptic curves $X$ with $h(x) \neq 0$ in (2.2) are also well known, see [3] and references within.

\section{Examples of auto Bäcklund transformations}

\subsection{Lagrange top}

Let us consider rotation of a rigid body around a fixed point in a homogeneous gravity field in the

Lagrange case. In terms of the Euler angles $\psi, \theta, \phi$, and momenta $p_{\psi}, p_{\theta}, p_{\phi}$ the Hamilton function looks like

$$
H=p_{\theta}^{2}+\frac{p_{\phi}^{2}+2 \cos \theta p_{\phi} p_{\psi}+p_{\psi}^{2}}{\sin ^{2} \theta}+\cos \theta .
$$


It is invariant with respect to canonical transformations of valence $c$

$$
\psi \rightarrow \tilde{\psi}=c \psi+a, \quad \phi \rightarrow \tilde{\phi}=c \phi+b, \quad \tilde{p}_{\psi}=p_{\psi}, \quad \tilde{p}_{\phi}=p_{\phi}
$$

For canonical transformation $(q, p) \rightarrow(\tilde{q}, \tilde{p})$ of valence $c$ the Jacobi matrix of transformation

$$
V=\left(\begin{array}{cc}
\frac{\partial \tilde{q}}{\partial q} & \frac{\partial \tilde{q}}{\partial p} \\
\frac{\partial \tilde{p}}{\partial q} & \frac{\partial \tilde{p}}{\partial p}
\end{array}\right)
$$

is a generalized symplectic matrix of valence $c$

$$
V^{\top} \Omega V=c \Omega, \quad \Omega=\left(\begin{array}{cc}
0 & I d \\
-I d & 0
\end{array}\right)
$$

see details in 7 .

Canonical transformation (3.12) is a symmetry and, according to Noether's theorem, this symmetry is related to integrals of motion

$$
A=p_{\psi}, \quad B=p_{\phi}
$$

We can also use this canonical transformation for construction of integrable discretization

$$
\psi_{k}=c_{k} \psi_{k-1}+a_{k}, \quad \phi_{k}=c_{k} \psi_{k-1}+b_{k}
$$

for dynamics of spin $\psi(t)$ and precession $\phi(t)$.

In order to describe similar canonical transformation, symmetry and discretization for nutation we can use the standard arithmetic on elliptic curves. Indeed, in [18] Lagrange noted that nutation $u=\cos \theta$ is the Weierstrass elliptic function $\wp(t)$ because Hamilton-Jacobi equation $H=E$

$$
\frac{v^{2}+A^{2}+2 u A B+B^{2}}{1-u^{2}}+u=E, \quad \text { where } \quad v=\sin \theta p_{\theta}
$$

defines the elliptic curve. It allows us to identify a tuple of integrals $H, p_{\psi}, p_{\phi}$ with a set of points $P=(\cos \theta, \sin \theta), P_{2}=\left(\psi, p_{\psi}\right.$ and $P_{3}=\left(\phi, p_{\phi}\right)$ on a direct product of the plane algebraic curves defined by separation relations (3.14) and (3.13).

For elliptic curve

$$
X: \quad y^{2}=f(x), \quad f(x)=a_{3} x^{3}+a_{2} x^{2}+a_{1} x+a_{0}
$$

the Jacobian of $X$ is isomorphic to the curve itself. So, let us consider points $P=(x, y), P^{\prime}=\left(x^{\prime}, y^{\prime}\right)$ and $P^{\prime \prime}=\left(x^{\prime \prime}, y^{\prime \prime}\right)$ on $X$ so that

$$
\text { Case 1: } P+P^{\prime}=P^{\prime \prime}, \quad \text { Case 2: } \quad[2] P=P^{\prime \prime}, \quad \text { Case } 3: \quad[3] P=P^{\prime \prime} \text {. }
$$

According to [10] coordinates of $P^{\prime \prime}$ are equal to

$$
\begin{aligned}
\text { 1. } x^{\prime \prime} & =-x-x^{\prime}-\frac{a_{2}}{a_{3}}+\frac{1}{a_{3}}\left(\frac{y-y^{\prime}}{x-x^{\prime}}\right)^{2}, & y^{\prime \prime} & =-\frac{x^{\prime \prime}-x^{\prime}}{x-x^{\prime}} y+\frac{x^{\prime \prime}-x}{x^{\prime}-x} y^{\prime}, \\
\text { 2. } x^{\prime \prime} & =-2 x-\frac{a_{2}}{a_{3}}+\frac{1}{a_{3}}\left(\frac{3 a_{3} x^{2}+2 a_{2} x+a_{1}}{2 y}\right)^{2}, & y^{\prime \prime} & =-y-\frac{\left(x^{\prime \prime}-x\right)\left(3 a_{3} x^{2}+2 a_{2} x+a_{1}\right)}{2 y}, \\
\text { 3. } x^{\prime \prime} & =-3 x-\frac{2 b_{1}}{b_{2}}-\frac{a_{3}}{b_{2}^{2}}, & y^{\prime \prime} & =b_{2} x^{\prime \prime 2}+b_{1} x^{\prime \prime}+b_{0},
\end{aligned}
$$

where $b_{k}$ are coefficients of the polynomial

$$
\mathcal{P}(z)=b_{2} z^{2}+b_{1} z+b_{0}=y+\frac{(z-x)\left(3 a_{3} x z+a_{2}(z+x)+a_{1}\right)}{2 y}-\frac{(z-x)^{2}\left(3 a_{3} x^{2}+2 a_{2} x+a_{1}\right)^{2}}{8 y^{3}}
$$


Substituting equation (3.14) for the Lagrange curve into these standard equations we obtain
1. $\quad \tilde{u}=-u-\lambda+H+\left(\frac{v-\mu}{u-\lambda}\right)^{2}$
$\tilde{v}=-\frac{\tilde{u}-\lambda}{u-\lambda} v+\frac{\tilde{u}-u}{\lambda-u} \mu$
2. $\tilde{u}=-2 u+H-\frac{\left(2 A B+2 H u-3 u^{2}+1\right)^{2}}{4 v^{2}}$,
$\tilde{v}=-v-\frac{(\tilde{u}-u)\left(2 A B+2 H u-3 u^{2}+1\right)}{2 v}$.

Here we put $(x, y)=(u, v),\left(x^{\prime \prime}, y^{\prime \prime}\right)=(\tilde{u}, \tilde{v})$ and $\left(x^{\prime}, y^{\prime}\right)=(\lambda, \mu)$ in order to distinguish points on the $(x, y)$-plane and coordinates on the phase space. In the third case we present polynomial $\mathcal{P}(z)$ only:

$$
\mathcal{P}(z)=v-\frac{(z-u)(2 A B+H(z+u)-3 z u+1)}{2 v}-\frac{(z-u)^{2}\left(2 A B+2 H u-3 u^{2}+1\right)^{2}}{8 v^{3}} .
$$

Proposition 1 Equations (3.16) determine canonical transformations $\left(\theta, p_{\theta}\right) \rightarrow\left(\tilde{\theta}, \tilde{p}_{\theta}\right)$ of valencies one, two and three, respectively. These canonical transformations preserve the form of Hamilton-Jacobi equation $H=E$, i.e. they are auto Bäcklund transformations for the Lagrange top.

The proof is a straightforward calculation in which we have taken into account that $\mu=\sqrt{f(\lambda)}$ is a function on $\theta, p_{\theta}$ and parameter $\lambda$.

\subsection{Hénon-Heiles system}

Let us take Hénon-Heiles system with Hamiltonians

$$
H_{1}=\frac{p_{1}^{2}+p_{2}^{2}}{4}-4 a q_{2}\left(q_{1}^{2}+2 q_{2}^{2}\right), \quad H_{2}=\frac{p_{1}\left(q_{1} p_{2}-q_{2} p_{1}\right)}{2}-a q_{1}^{2}\left(q_{1}^{2}+4 q_{2}^{2}\right)
$$

separable in parabolic coordinates on the plane

$$
u_{1}=q_{2}-\sqrt{q_{1}^{2}+q_{2}^{2}}, \quad u_{2}=q_{2}+\sqrt{q_{1}^{2}+q_{2}^{2}} .
$$

Standard momenta associated with parabolic coordinates $u_{1,2}$ are equal to

$$
p_{u_{1}}=\frac{p_{2}}{2}-\frac{p_{1}\left(q_{2}+\sqrt{q_{1}^{2}+q_{2}^{2}}\right)}{2 q_{1}}, \quad p_{u_{2}}=\frac{p_{2}}{2}-\frac{p_{1}\left(q_{2}-\sqrt{q_{1}^{2}+q_{2}^{2}}\right)}{2 q_{1}} .
$$

To describe evolution of $u_{1,2}$ with respect to $H_{1,2}$ we use the canonical Poisson bracket

$$
\left\{q_{i}, p_{j}\right\}=\delta_{i j}, \quad\left\{q_{1}, q_{2}\right\}=\left\{p_{1}, p_{2}\right\}=0, \quad\left\{u_{i}, p_{u_{j}}\right\}=\delta_{i j}, \quad\left\{u_{1}, u_{2}\right\}=\left\{p_{u_{1}}, p_{u_{2}}\right\}=0 .
$$

and expressions for $H_{1,2}$

$$
H_{1}=\frac{p_{u_{1}}^{2} u_{1}-p_{u_{2}}^{2} u_{2}}{u_{1}-u_{2}}-a\left(u_{1}+u_{2}\right)\left(u_{1}^{2}+u_{2}^{2}\right), \quad H_{2}=\frac{u_{1} u_{2}\left(p_{u_{1}}^{2}-p_{u_{2}}^{2}\right)}{u_{2}-u_{1}}+a u_{1} u_{2}\left(u_{1}^{2}+u_{1} u_{2}+u_{2}^{2}\right)
$$

to obtain

$$
\frac{d u_{1}}{d t_{1}}=\left\{u_{1}, H_{1}\right\}=\frac{2 p_{u_{1}} u_{1}}{u_{1}-u_{2}}, \quad \frac{d u_{2}}{d t_{1}}=\left\{u_{2}, H_{1}\right\}=\frac{2 p_{u_{2}} u_{2}}{u_{2}-u_{1}}
$$

and

$$
\frac{d u_{1}}{d t_{2}}=\left\{u_{1}, H_{2}\right\}=\frac{2 u_{1} u_{2} p_{u_{1}}}{u_{2}-u_{1}}, \quad \frac{d u_{2}}{d t_{2}}=\left\{u_{2}, H_{2}\right\}=\frac{2 u_{1} u_{2} p_{u_{2}}}{u_{1}-u_{2}} .
$$

Using Hamilton-Jacobi equations $H_{1,2}=\alpha_{1,2}$ we can prove that these variables satisfy to the following separated relations

$$
\left(u_{i} p_{u_{i}}\right)^{2}=u_{i}\left(a u_{i}^{4}+\alpha_{1} u_{i}+\alpha_{2}\right), \quad i=1,2 .
$$

Expressions (3.21, 3.22) and (3.23) yield standard Abel quadratures

$$
\frac{d u_{1}}{\sqrt{f\left(u_{1}\right)}}+\frac{d u_{2}}{\sqrt{f\left(u_{2}\right)}}=2 d t_{2}, \quad \frac{u_{1} d u_{1}}{\sqrt{f\left(u_{1}\right)}}+\frac{u_{2} d u_{2}}{\sqrt{f\left(u_{2}\right)}}=2 d t_{1},
$$


on hyperelliptic curve $X$ of genus two defined by equation

$$
X: \quad y^{2}=f(x), \quad f(x)=x\left(a x^{4}+\alpha_{1} x+\alpha_{2}\right) .
$$

Suppose that transformation of variables

$$
\left(q_{1}, q_{2}, p_{1}, p_{2}\right) \rightarrow\left(\tilde{q}_{1}, \tilde{q}_{2}, \tilde{p}_{1}, \tilde{p}_{2}\right)
$$

preserves Hamilton equations $(3.21+3.22)$ and the form of Hamiltonians (3.20). It means that new parabolic coordinates $\tilde{u}_{1,2}=\tilde{q}_{2} \pm \sqrt{\tilde{q}_{1}^{2}+\tilde{q}_{2}^{2}}$ satisfy to the same equations

$$
\frac{d \tilde{u}_{1}}{\sqrt{f\left(\tilde{u}_{1}\right)}}+\frac{d \tilde{u}_{2}}{\sqrt{f\left(\tilde{u}_{2}\right)}}=2 d t_{2}, \quad \frac{\tilde{u}_{1} d \tilde{u}_{1}}{\sqrt{f\left(\tilde{u}_{1}\right)}}+\frac{\tilde{u}_{2} d \tilde{u}_{2}}{\sqrt{f\left(\tilde{u}_{2}\right)}}=2 d t_{1} .
$$

Subtracting (3.27) from (3.24) one gets Abel differential equations

$$
\begin{aligned}
& \omega_{1}\left(x_{1}, y_{1}\right)+\omega_{1}\left(x_{2}, y_{2}\right)+\omega_{1}\left(x_{1}^{\prime \prime}, y_{1}^{\prime \prime}\right)+\omega_{1}\left(x_{2}^{\prime \prime}, y_{2}^{\prime \prime}\right)=0, \\
& \omega_{2}\left(x_{1}, y_{1}\right)+\omega_{2}\left(x_{2}, y_{2}\right)+\omega_{2}\left(x_{2}^{\prime \prime}, y_{1}^{\prime \prime}\right)+\omega_{2}\left(x_{2}^{\prime \prime}, y_{2}^{\prime \prime}\right)=0,
\end{aligned}
$$

where

$$
x_{1,2}=u_{1,2}, \quad y_{1,2}=u_{1,2} p_{u_{1,2}}, \quad x_{1,2}^{\prime \prime}=\tilde{u}_{1,2}, \quad y_{1,2}^{\prime \prime}=-\tilde{u}_{1,2} \tilde{p}_{u_{1,2}}
$$

and $\omega_{1,2}$ form a base of holomorphic differentials on hyperelliptic curve $X$ of genus $g=2$

$$
\omega_{1}(x, y)=\frac{d x}{y}, \quad \omega_{2}(x, y)=\frac{x d x}{y} .
$$

Solutions of the Abel equations form so-called intersection divisor of two plane curves $X$ and $Y$ [1, 15], that allows us directly apply cryptographic protocols based on arithmetic of divisor to construction of canonical transformation (3.26) on the phase space.

Let us suppose that generic points $P_{1}=\left(x_{1}, y_{1}\right)$ and $P_{2}=\left(x_{2}, y_{2}\right)$ form divisor $D$ (message) in (2.4), whereas points $P_{1,2}^{\prime \prime}=\left(x_{1,2}^{\prime \prime}, y_{1,2}^{\prime \prime}\right)$ belong to support of resulting divisor $D^{\prime \prime}$ (cryptogram). Canonical transformations (3.26) associated with arithmetic operations (2.4) are completely defined by coefficients $b_{k}$ of polynomial $\mathcal{P}$ (2.11). In the first case, these coefficients $b_{k}$ are defined by

$$
\begin{aligned}
A b_{0}= & q_{1} x_{1}^{\prime} x_{2}^{\prime}\left(x_{1}^{\prime}-x_{2}^{\prime}\right)\left(p_{1}\left(q_{1}^{2}+4 q_{2}^{2}-2 q_{2}\left(x_{1}^{\prime}+x_{2}^{\prime}\right)+x_{1}^{\prime} x_{2}^{\prime}\right)+q_{1} p_{2}\left(x_{1}^{\prime}+x_{2}^{\prime}+2 q_{2}\right)\right) \\
- & 2 q_{1}^{2} x_{2}^{\prime}\left(q_{1}^{2}+2 q_{2} x_{2}^{\prime}-{x_{2}^{\prime}}^{2}\right) y_{1}^{\prime}+2 q_{1}^{2} x_{1}^{\prime}\left(q_{1}^{2}+2 q_{2} x_{1}^{\prime}-x_{2}^{\prime 2}\right) y_{2}^{\prime} ; \\
A b_{1}= & \left(x_{2}^{\prime}-x_{1}^{\prime}\right)\left(q_{1} p_{1}\left(\left(q_{1}^{2}+4 q_{2}^{2}\right)\left(x_{1}^{\prime}+x_{2}^{\prime}\right)-2 q_{2}\left(x_{1}^{\prime 2}+x_{1}^{\prime} x_{2}^{\prime}+x_{2}^{\prime 2}\right)\right)\right. \\
& \left.\quad-p_{2}\left(2 q_{1}^{2} q_{2}\left(x_{1}^{\prime}+x_{2}^{\prime}\right)-q_{1}^{2}\left(x_{1}^{\prime 2}+x_{1}^{\prime} x_{2}^{\prime}+x_{2}^{\prime 2}\right)+x_{1}^{\prime 2} x_{2}^{\prime 2}\right)\right) \\
+ & 2\left(q_{1}^{2}-2 q_{2} x_{2}^{\prime}\right)\left(q_{1}^{2}+2 q_{2} x_{2}^{\prime}-x_{2}^{\prime 2}\right) y_{1}^{\prime}-2\left(q_{1}^{2}-2 q_{2} x_{1}^{\prime}\right)\left(q_{1}^{2}+2 q_{2} x_{1}^{\prime}-x_{1}^{\prime 2}\right) y_{2}^{\prime}, \\
A b_{2}= & \left(x_{1}^{\prime}-x_{2}^{\prime}\right)\left(q_{1} p_{1}\left(q_{1}^{2}+4 q_{2}^{2}-x_{1}^{\prime 2}-x_{1}^{\prime} x_{2}^{\prime}-x_{2}^{\prime 2}\right)-\left(2 q_{1}^{2} q_{2}+\left(x_{1}^{\prime}+x_{2}^{\prime}\right) x_{1}^{\prime} x_{2}^{\prime}\right) p_{2}\right) \\
+ & 2\left(2 q_{2}+x_{2}^{\prime}\right)\left(q_{1}^{2}+2 q_{2} x_{2}^{\prime}-x_{2}^{\prime 2}\right) y_{1}^{\prime}-2\left(2 q_{2}+x_{1}^{\prime}\right)\left(q_{1}^{2}+2 q_{2} x_{1}^{\prime}-x_{1}^{\prime 2}\right) y_{2}^{\prime}, \\
A b_{3}= & \left(x_{2}^{\prime}-x_{1}^{\prime}\right)\left(q_{1} p_{1}\left(2 q_{2}-x_{1}^{\prime}-x_{2}^{\prime}\right)-\left(q_{1}^{2}+x_{1}^{\prime} x_{2}^{\prime}\right) p_{2}\right)-2\left(q_{1}^{2}+2 q_{2} x_{2}^{\prime}-x_{2}^{\prime 2}\right) y_{1}^{\prime} \\
- & 2\left(q_{1}^{2}+2 q_{2} x_{1}^{\prime}-x_{1}^{\prime 2}\right) y_{2}^{\prime},
\end{aligned}
$$

where

$$
A=2\left(x_{1}^{\prime}-x_{2}^{\prime}\right)\left(q_{1}^{2}+2 q_{2} x_{1}^{\prime}-x_{2}^{\prime 2}\right)\left(q_{1}^{2}+2 q_{2} x_{2}^{\prime}-x_{2}^{\prime 2}\right) .
$$


Substituting these coefficients into the following expressions one gets explicit formulae for the new variables

$$
\begin{aligned}
& \tilde{q}_{2}=-q_{2}-\frac{x_{1}^{\prime}+x_{2}^{\prime}}{2}-\frac{b_{2}}{b_{3}}+\frac{a}{2 b_{3}^{2}} \\
& \tilde{q}_{1}^{2}=-q_{1}^{2}+2 \tilde{q}_{2}\left(2 q_{2}+x_{1}^{\prime}+x_{2}^{\prime}\right)+2 q_{2}\left(x_{1}^{\prime}+x_{2}^{\prime}\right)+x_{1}^{\prime} x_{2}^{\prime}-\frac{2 b_{1}}{b_{3}}-\frac{b_{2}^{2}}{b_{3}^{2}} \\
& \tilde{p}_{1}=-\frac{2 b_{0}}{\tilde{q}_{1}}-4 \tilde{q}_{1} \tilde{q}_{2} b_{3}-2 \tilde{q}_{1} b_{2}, \quad \tilde{p}_{2}=-2\left(\tilde{q}_{1}^{2}+4 \tilde{q}_{2}^{2}\right) b_{3}-4 \tilde{q}_{2} b_{2}-2 b_{1} .
\end{aligned}
$$

In Case 2 we have

$$
\begin{aligned}
B b_{0} & =-8 a q_{1}^{4}\left(2 p_{1} q_{1} q_{2}-p 2 q_{1}^{2}-2 p_{2} q_{2}^{2}\right)+q_{1}^{2} p_{1}\left(p_{1}^{2} q_{1}+2 p_{1} p_{2} q_{2}-2 p 2^{2} q_{1}\right), \\
B b_{1} & =-8 a q_{1}^{2}\left(p_{1} q_{1}^{3}+10 p_{1} q_{1} q_{2}^{2}-4 p_{2} q_{1}^{2} q_{2}-4 p_{2} q_{2}^{3}\right)-2 p_{1}^{3} q_{1} q_{2}+3 p_{1}^{2} q_{1}^{2} p_{2}-4 p_{1}^{2} p_{2} q_{2}^{2} \\
& +4 p_{1} p_{2}^{2} q_{1} q_{2}-2 p_{2}^{3} q_{1}^{2}, \\
B b_{2} & =-8 a q_{1}\left(12 p_{1} q_{2}^{3}+p_{2} q_{1}^{3}-2 p_{2} q_{1} q_{2}^{2}\right)+p_{1}^{2}\left(p_{1} q_{1}+4 p_{2} q_{2}\right), \\
B b_{3} & =8 a q_{1}\left(p_{1} q_{1}^{2}+6 p_{1} q_{2}^{2}-2 p_{2} q_{1} q_{2}\right)-p_{2} p_{1}^{2}, \quad B=4 q_{1}\left(p_{1}^{2} q_{1}+2 p_{1} p_{2} q_{2}-p_{2}^{2} q_{1}\right)
\end{aligned}
$$

so that

$$
\begin{aligned}
\tilde{q}_{2}= & -2 q_{2}+\frac{1}{\left(8 q_{1}\left(p_{1} q_{1}^{2}+6 p_{1} q_{2}^{2}-2 p_{2} q_{1} q_{2}\right) a-p_{2} p_{1}^{2}\right)^{2}} \\
\times & \left(64 q_{1}^{2}\left(12 p_{1} q_{2}^{3}+p_{2} q_{1}^{3}-2 p_{2} q_{1} q_{2}^{2}\right)\left(p_{1} q_{1}^{2}+6 p_{1} q_{2}^{2}-2 p_{2} q_{1} q_{2}\right) a^{2}+p_{1}^{4} p_{2}\left(p_{1} q_{1}+4 p_{2} q_{2}\right)\right. \\
& \left.-8 a q_{1}\left(6 p_{1}^{4} q_{1} q_{2}^{2}-2 p_{1}^{3} p_{2} q_{1}^{2} q_{2}+36 p_{1}^{3} p_{2} q_{2}^{3}+3 p_{1}^{2} p_{2}^{2} q_{1}^{3}-14 p_{1}^{2} p_{2}^{2} q_{1} q_{2}^{2}+4 p_{1} p_{2}^{3} q_{1}^{2} q_{2}-p_{2}^{4} q_{1}^{3}\right)\right) \\
\tilde{q}_{1}^{2}= & -\left(\frac{8 a q_{1}^{2}\left(2 p_{1} q_{1} q_{2}-p_{2} q_{1}^{2}-2 p_{2} q_{2}^{2}\right)-p_{1}\left(p_{1}^{2} q_{1}+2 p_{1} p_{2} q_{2}-2 p_{2}^{2} q_{1}\right.}{8 a q_{1}\left(p_{1} q_{1}^{2}+6 p_{1} q_{2}^{2}-2 p_{2} q_{1} q_{2}\right)-p_{2} p_{1}^{2}}\right)^{2} \\
\tilde{q}_{1} \tilde{p}_{1}= & -2 b_{0}-2 \tilde{q}_{1}^{2}\left(b_{2}+2 \tilde{q}_{2} b_{3}\right), \quad \tilde{p}_{2}=-2\left(\tilde{q}_{1}^{2}+4 \tilde{q}_{2}^{2}\right) b_{3}-4 \tilde{q}_{2} b_{2}-2 b_{1}
\end{aligned}
$$

In Case 3 coefficients are equal to

$$
\begin{aligned}
& b_{0}=\frac{\left(x_{1}^{\prime}\left(2 p_{1} q_{2} x_{1}^{\prime}-p_{2} q_{1}\right) p_{1}{x_{1}^{\prime}}^{2}+2 q_{1} y_{1}^{\prime}\right) q_{1}}{2\left(q_{1}^{2}+2 q_{2} x_{1}^{\prime}-x_{1}^{\prime 2}\right)} \\
& b_{1}=-\frac{2 p_{1} q_{1} q_{2}-p_{2}\left(q_{1}^{2}-x_{1}^{\prime 2}\right)-4 q_{2} y_{1}^{\prime}}{2\left(q_{1}^{2}+2 q_{2} x_{1}^{\prime}-{x_{1}^{\prime}}^{2}\right)}, \quad b_{2}=\frac{p_{1} q_{1}+p_{2} x_{1}^{\prime}-2 y_{1}^{\prime}}{2\left(q_{1}^{2}+2 q_{2} x_{1}^{\prime}-{x_{1}^{\prime}}^{2}\right)},
\end{aligned}
$$


so that

$$
\begin{aligned}
\tilde{q}_{2} & =-q_{2}-\frac{x_{1}^{\prime}}{2}+\frac{b_{2}^{2}}{2 a}=-q_{2}-\frac{x_{1}^{\prime}}{2}+\frac{\left(p_{1} q_{1}+p_{2} x_{1}^{\prime}-2 y_{1}^{\prime}\right)^{2}}{8 a\left(q_{1}^{2}+2 q_{2} x_{1}^{\prime}-x_{1}^{\prime 2}\right)^{2}} \\
\tilde{q}_{1}^{2} & =-q_{1}^{2}+2 \tilde{q}_{2}\left(2 q_{2}+x_{1}^{\prime}\right)+2 q_{2} x_{1}^{\prime}+\frac{2 b_{1} b_{2}}{a} \\
& =-q_{1}^{2}+2 \tilde{q}_{2}\left(2 q_{2}+x_{1}^{\prime}\right)+2 q_{2} x_{1}^{\prime}-\frac{\left(p_{1} q_{1}+p_{2} x_{1}^{\prime}-2 y_{1}^{\prime}\right)\left(2 p_{1} q_{1} q_{2}-p_{2} q_{1}^{2}+p_{2} x_{1}^{\prime 2}-4 q_{2} y_{1}^{\prime}\right)}{2 a\left(q_{1}^{2}+2 q_{2} x_{1}^{\prime}-x_{1}^{\prime 2}\right)^{2}}, \\
\tilde{p}_{2} & =-4 \tilde{q}_{2} b_{2}-2 b_{1}=-p_{2}-\frac{2\left(q_{2}-\tilde{q}_{2}\right)\left(2 y_{1}^{\prime}-p_{1} q_{1}-p_{2} x_{1}^{\prime}\right)}{q_{1}^{2}+2 q_{2} x_{1}^{\prime}-x_{1}^{\prime 2}} \\
\tilde{q}_{1} \tilde{p}_{1} & =-2 b_{0}-2 q_{1}^{2} b_{2}=-q_{2} p_{2}-\frac{\left(q_{1}^{2}-\tilde{q}_{2}^{2}\right)\left(2 y_{1}^{\prime}-p_{1} q_{1}-p_{2} x_{1}^{\prime}\right)}{q_{1}^{2}+2 q_{2} x_{1}^{\prime}-x_{1}^{\prime 2}} .
\end{aligned}
$$

These explicit formulae for $\tilde{q}_{1,2}$ and $\tilde{p}_{1,2}$ can be easily obtained using any modern computer algebra system.

We present these bulky expressions here only so that any reader can verify that these transformations $(q, p) \rightarrow(\tilde{q}, \tilde{p})$ are different, i.e. cannot be obtained from each other for special values of parameters, and that these transformations have the following properties.

Proposition 2 Equations (3.29(3.30) and (3.32) determine canonical transformations (3.26) on $T^{*} \mathbb{R}^{2}$ of valencies one and two for which original Poisson bracket (3.19) has the following form in new variables

$$
\begin{array}{rlr}
1,3 . & \left\{\tilde{q}_{i}, \tilde{p}_{j}\right\}=\delta_{i, j}, \quad\left\{\tilde{q}_{1}, \tilde{q}_{2}\right\}=\left\{\tilde{p}_{1}, \tilde{p}_{2}\right\}=0, \\
2 . & \left\{\tilde{q}_{i}, \tilde{p}_{j}\right\}=2 \delta_{i, j}, \quad\left\{\tilde{q}_{1}, \tilde{q}_{2}\right\}=\left\{\tilde{p}_{1}, \tilde{p}_{2}\right\}=0,
\end{array}
$$

respectively. These canonical transformations preserve the form of integrals of motion (3.17), i.e. they are auto Bäcklund transformations in the Toda-Wadati sense [24].

The proof is a straightforward calculation.

The fact most interesting to us is that obtained cryptograms are new canonical variables on the original phase space, which can be used for construction of new integrable systems in the framework of the Jacobi method.

For instance, let us suppose that divisor $D^{\prime}$ (secret key) consists of ramification point $P_{0}=(0,0)$ and point $P_{\infty}$. In this case cryptogram, i.e canonical variables $\tilde{u}_{1,2}, \tilde{p}_{u_{1,2}}$ are defined by equations (2.8) and (2.10) for $x_{k}^{\prime \prime}=\tilde{u}_{k}$ and $y_{k}^{\prime \prime}=\tilde{u}_{k} \tilde{p}_{u_{k}}$ :

$$
\begin{aligned}
& \tilde{u}_{1}+\tilde{u}_{2}=-u_{1}-u_{2}+\frac{b_{2}^{2}}{a}, \quad \tilde{u}_{1} \tilde{u}_{2}=-\frac{2 b_{1} b_{2}}{a}-\left(u_{1}+u_{2}\right)\left(\tilde{u}_{1}+\tilde{u}_{2}\right)-u_{1} u_{2}, \\
& \tilde{p}_{u_{1}}=-\frac{b_{2} \tilde{u}_{1}^{2}+b_{1} \tilde{u}_{1}+b_{0}}{\tilde{u}_{1}}, \quad \tilde{p}_{u_{2}}=-\frac{b_{2} \tilde{u}_{2}^{2}+b_{1} \tilde{u}_{2}+b_{0}}{\tilde{u}_{2}},
\end{aligned}
$$

where coefficients $b_{k}$ are given by (3.31). Substituting $y=\tilde{p}_{1,2}$ and $x=\tilde{u}_{1,2}$ into the separated relation

$$
\tilde{X}: \quad\left(y^{2}-a x^{3}-\tilde{H}_{1}-\tilde{H}_{2}\right)\left(y^{2}-a x^{3}-\tilde{H}_{1}+\tilde{H}_{2}\right)+a b x+a c y=0,
$$

which defines genus three hyperelliptic curve $\tilde{X}$, and solving the resulting equations with respect to $\tilde{H}_{1,2}$, one gets Hamiltonian

$$
\tilde{H}_{1}=\frac{p_{1}^{2}}{8}+\frac{p_{2}^{2}}{4}-a q_{2}\left(3 q_{1}^{2}+8 q_{2}^{2}\right)+\frac{b}{2 q_{1}^{2}}-\frac{c p_{1}}{q_{1}^{3}} .
$$

We can identify this Hamiltonian with well-known second integrable Hénon-Heiles system with quartic additional integral $H_{2}$ 25, 27.

According [26, 28, 29, 30, 31] we can use these cryptographic protocols in order to get new integrable systems on the plane, sphere and ellipsoid with polynomial integrals of motion of sixth, fourth and third order in momenta.

The work was supported by the Russian Science Foundation (project 15-12-20035). 


\section{References}

[1] Abel N. H., Mémoire sure une propriété générale d'une class très éntendue des fonctions transcendantes, Euvres complétes, Tom I, Grondahl Son, Christiania (1881), pages 145-211.

[2] Cantor D.G., Computing in the Jacobian of a hyperelliptic curve, Mathematics of computation, (1987), v.48, n.177, pp.95-101.

[3] Costello C., Lauter K., Group law computations on Jacobians of hyperelliptic curves, In: Miri, A., Vaudenay, S. (eds.) SAC 2011, LNCS, vol. 7118, pp. 92-117, Springer, Heidelberg (2012).

[4] Costello C., A gentle introduction to isogeny-based cryptography, Tutorial at SPACE 2016. December 15, 2016. CRRao AIMSCS, Hyderabad, India 2016.

[5] Eisenbud D., Harris J., 3264 and all that: A second course in algebraic geometry, pp. 632. Cambridge University Press, 2016.

[6] Fedorov Yu., Discrete versions of some algebraic integrable systems related to generalized Jacobians, in SIDE III: Symmetries and Integrability of Difference Equations, (Sabaudia, 1998), CRM Proc. Lecture Notes, vol.25, Amer. Math. Soc., Providence, RI, (2000), pp.147-160.

[7] Gantmacher F., Lectures in Analytical Mechanics, Mir Publishers, Moscow, 1975.

[8] Gaudry P., Harley R., Counting points on hyperelliptic curves over finite fields, In W. Bosma, editor, ANTS, vol.1838 of Lecture Notes in Computer Science, Springer, (2000), pp.313-332.

[9] Hartshorne R., Algebraic geometry, volume 52 of Graduate Texts in Mathematics, Springer-Verlag, New York, 1977.

[10] Handbook of Elliptic and Hyperelliptic Curve Cryptography, editors H. Cohen and G. Frey, Chapman and Hall/CRC, 2006.

[11] Harley R., Fast arithmetic on genus two curves, at http://cristal.inria.fr/ ^ harley/hyper/, (2000).

[12] Hone A.N.W., Kuznetsov V.B., Ragnisco O., Bäcklund transformations for many-body systems related to KdV, J. Phys. A: Math. Gen., (1999), v.32, p.L299-L306.

[13] Inoue R., Konishi Y., Yamazaki T., Jacobian variety and integrable system - after Mumford, Beauville and Vanhaecke, Journal of Geometry and Physics, (2007), v. 57, n.3, p. 815-831.

[14] Katagi M., Kitamura I., Akishita T., Takagi T,, Novel efficient implementations of hyperelliptic curve cryptosystems using degenerate divisors, in: Information Security Applications: 5th International Workshop, WISA 2004, Jeju Island, Korea, August 23-25, 2004, Revised Selected Papers, editirs Lim C.H. and Yung M., 2005, Springer Berlin Heidelberg, pp. 345-359.

[15] Kleiman S.L., The Picard scheme, Fundamental Algebraic Geometry, Math. Surveys Monogr., v.123, Amer. Math. Soc., Providence, RI (2005), pp.235-321.

[16] Kuznetsov V.B., Vanhaecke P., Bäcklund transformations for finite-dimensional integrable systems: a geometric approach, Journal of Geometry and Physics, (2002), vol.44, n.1, pp.1-40.

[17] Kuznetsov V. B., Petrera M., Ragnisco O., Separation of variables and Bäcklund transformations for the symmetric Lagrange top, J. Phys. A, (2004), v.37, pp. 8495-8512.

[18] Lagrange J.L., Mécanique Analytique, 1788, in Evres de Lagrange, tome XII, Gauthier-Villars, 1889.

[19] Lange T., Efficient arithmetic on hyperelliptic curves, PhD thesis, Universität-Gesamthochschule Essen, 2001.

[20] Miret J.M., Moreno R., Pujolàs J., Rio A., Halving for the 2-Sylow subgroup of genus 2 curves over binary fields, Finite Fields and Their Applications, (2009), v.15, n. 5, p.569-579.

[21] D. Mumford D., Tata Lectures on Theta II, Birkhäuser, 1984. 
[22] Sklyanin E.K., Bäcklund transformations and Baxter's Q-operator, in Integrable Systems: from Classical to Quantum (1999, Montreal), CRM Proc. Lecture Notes, vol. 26, Amer. Math. Soc., Providence, RI, (2000), pp. 227-250.

[23] Sutherland A.V., Fast Jacobian arithmetic for hyperelliptic curves of genus 3, arXiv: 1607.08602, (2016).

[24] M. Toda, M. Wadati, A canonical transformation for the exponential lattice, J. Phys. Soc. Japan, (1975), 39, 1204-1211.

[25] Tsiganov A.V., Simultaneous separation for the Neumann and Chaplygin systems, Reg. Chaotic Dyn., (2015), v.20, pp.74-93.

[26] Tsiganov A.V., On the Chaplygin system on the sphere with velocity dependent potential, J. Geom. Phys., (2015), v.92, pp.94-99.

[27] Tsiganov A.V., On auto and hetero Bäcklund transformations for the Hénon-Heiles systems, Phys. Letters A, (2015), v.379, pp.2903-2907.

[28] Tsiganov A. V. Bäcklund transformations for the nonholonomic Veselova system, Regular and Chaotic Dynamics, (2017), v. 22(2), pp. 163-179.

[29] Tsiganov A.V. Integrable discretization and deformation of the nonholonomic Chaplygin ball, Regular and Chaotic Dynamics, (2017), v.22(4), pp. 353-367.

[30] Tsiganov A.V. New bi-Hamiltonian systems on the plane, Journal of Mathematical Physics, (2017), v.58, 062901.

[31] Tsiganov A.V. Bäcklund transformations for the Jacobi system on an ellipsoid, Theoretical and Mathematical Physics, (2017), v. 192(3), pp.1204-1218.

[32] Vanhaecke P., Integrable systems in the realm of algebraic geometry, Lecture Notes in Mathematics, vol. 1638, (2001). 